\title{
Sexual imprinting as a two-stage process: mechanisms of information storage and stabilization
}

\author{
SABINE OETTING, EKKEHARD PRÖVE\& HANS-JOACHIM BISCHOF* \\ $L$ ehrstuhl für $V$ erhaltensforschung, F akultät Biologie, U niversität Bielefeld, P ostfach 100131, \\ 33501 Bielefeld, Germany
}

( Received 7 J uly 1994; initial acceptance 18 A ugust 1994;

final acceptance 4 N ovember 1994; M S. number: 4691)

\begin{abstract}
A bstract. Sexual imprinting occurs in two stages, an acquisition phase when the birds learn about their social environment, and a consolidation process where the acquired information is linked to sexual behaviour and stabilized. Some of the factors that may influence acquisition and consolidation, respectively, were examined. Zebra finch, Taeniopygia guttata, males were reared by Bengalese finch, L onchura striata, foster parents until 40 days of age, then isolated for 60 days, and exposed to a zebra finch female for $1 \mathrm{~h}$ at day 100 . Thereafter, the birds were isolated for 3 days and then exposed to a Bengalese finch female for another $1 \mathrm{~h}$. In subsequent preference tests, the preference scores of the birds were correlated with how much they were fed by their foster parents. The influence of the first exposure to a zebra finch female correlated positively with the level of arousal of the experimental males, as indicated by measurements of displacement activities and corticosterone levels. When first exposed to a female as adults, the experimental males could learn features of a potential sexual partner and, simultaneously, the acquired preference was consolidated. Consolidation, however, was independent of the appearance of the female: after having a 24-h access to a nestbox on day 99 , the experimental males developed an exclusive preference for Bengalese finches. These results confirm that acquisition and consolidation are different processes which can be separated at least by the appropriate experimental design.

(C) 1995 The A ssociation for the Study of A nimal Behaviour
\end{abstract}

Traditionally, sexual imprinting has been defined as a learning process by which a young bird learns the characteristics of its species, which enables it to find a sexual partner as an adult (Bateson 1966; Immelmann 1972a, b; H ess 1973). F or the zebra finch, T aeniopygia guttata castanotis, the sensitive period for sexual imprinting begins after the eyes open and comes to its close between days 30 and 40 post-hatch, although under certain circumstances it can be prolonged (for a review see Immelmann 1985). Because the young bird is not sexually mature at that time and thus no sexual behaviour can be observed, one has to ask how the bird 'knows' what the appropriate features of a future sexual partner may be. It is possible that some preexisting knowledge guides the acquisition of the appropriate features. There have been several claims of such an 'own species bias' (Immelmann 1972a, b) but severe arguments have been raised against such a view (K ruijt \& M eeuwissen 1993).

*To whom all correspondence should be addressed.
Immelmann et al. (1991) and Kruijt \& M eeuwissen (1991) recently published results which suggest a more plausible explanation. According to their experiments, sexual imprinting may be a two-stage process. In the course of the 'classical' sensitive phase in early development, the birds establish a social bond to their parents. U nder normal circumstances, this narrowing of social preference to the parent species guides the young male zebra finches in their first courtship attempts when they are sexually mature. By these first courtship events, the preference for the parent species is linked to sexual behaviour and is stabilized. If, however, they can court only females that do not resemble the parent species, modification of the preference is possible. In other words, the social preference established in early development is not transferred to sexual preference: zebra finch males reared by Bengalese finch, L onchura striata, foster parents sometimes switch their preference to zebra finches if the first courtship event is with a zebra finch female (Bischof \& Clayton 1991; Immelmann et al. 1991; K ruijt \& 
Experiment II only: nestbox/rear of the nestbox on day 99

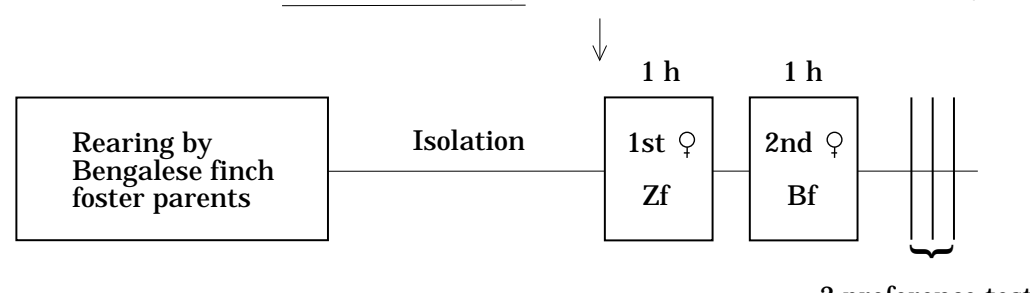

3 preference tests

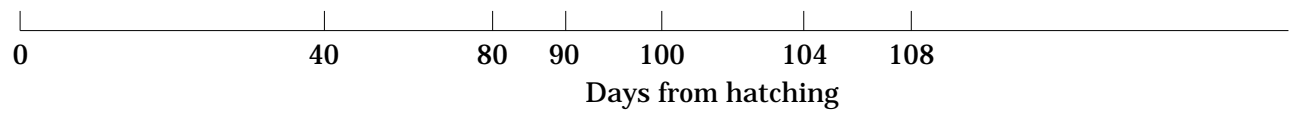

Experiment I only:

Blood samples

Figure 1. Experimental design for experiments I and II. Zf: Zebra finch; Bf: Bengalese finch. F or further explanations see text.

M eeuwissen 1991, 1993). It is therefore possible to distinguish two successive stages of sexual imprinting, an acquisition phase, which is identical to the 'classical' sensitive phase, and a process of consolidation, which occurs when the male zebra finch is sexually mature (Bischof 1994).

A lthough this idea of two separate stages is well established by several independent studies, not much is known about the factors affecting the establishment of a given preference. A s mentioned above, not all the zebra finch males that were reared by Bengalese finch foster parents and, as adults, had their first courtship experience with a zebra finch female, switched their preference from Bengalese finch to zebra finch females. Bischof \& Clayton (1991) provided the first evidence that social interactions in both the acquisition phase and the consolidation process may play a role in the decision as to which species was to be preferred. They showed that young males were more likely to prefer the Bengalese finch foster species if they were fed more by their foster parents, and that frequent courtship behaviour during the first courtship event with a zebra finch female made a preference for zebra finch females more likely.

On the other hand, there is some evidence that physical contact with the social or sexual partner is not crucial for acquisition and consolidation of preferences. In both of the basic studies (Immelmann et al. 1991; K ruijt \& M eeuwissen
1991) consolidation occurred when the females were separated by wire mesh, and $\mathrm{K}$ ruijt \& M eeuwissen (1991), with the same experimental design, also showed that the initially acquired preference can be modified. Instead of direct interaction, the enhancement of arousal induced by the presence of the female may play an important role. This idea was supported by physiological studies (Bischof \& Herrmann 1986, 1988) which indicated that the presence of a female after a period of isolation increases the male's arousal level.

Our aim in our first experiment was two-fold: first, to re-examine and confirm the amplifying effect of feeding on learning the parents' features; and second, to test whether the level of arousal at the time of the adult male's first exposure to a female correlates with consolidation of acquired preferences. In our second experiment we tested whether the exposure to a female is necessary for consolidation to occur, or whether consolidation also happens if the bird comes into another sexually arousing context.

\section{EXPERIM ENT I}

\section{$M$ ethods}

Figure 1 shows the rearing and testing conditions. Eggs of zebra finch pairs were transferred to, and reared by, Bengalese finch foster parents, 
whose own eggs were removed. In each case, a whole clutch was transferred, and so the number of siblings varied between zero and four. Each breeding pair was housed in an extra cage $(80 \times 40 \times 30 \mathrm{~cm})$. At 40 days of age, the young birds were isolated and placed in single cages $(40 \times 30 \times 30 \mathrm{~cm})$ in another room where they could hear, but not see, other zebra finch males and females. Food and water were available ad libitum except for $5 \mathrm{~h}$ preceding observations (see below). Food consisted of dry and sprouted seed as well as occasional fresh greens. A multivitamin preparation was given into the water two or three times weekly. Pairs rearing nestlings also got a mixture of cooked hen's eggs, minerals and vitamins. All housing rooms received daylight and supplementary artificial lighting, so that the animals received $14 \mathrm{~h}$ of light and $10 \mathrm{~h}$ of darkness. The temperature in the housing rooms was held around $24^{\circ} \mathrm{C}$.

A first group of 20 males was exposed to a zebra finch female for $1 \mathrm{~h}$ at 100 days of age. A fter 3 days of isolation, at the age of 104 days, the males were exposed for $1 \mathrm{~h}$ to a Bengalese finch female, at the same hour of the day as the first exposure. For both exposures, each zebra finch male was transferred in his home cage into a separate room, and the female was put into this cage. A fter another 3 days of isolation the birds were tested three times, once a day, on successive days in a preference test lasting $20 \mathrm{~min}$. In these tests, the males could choose between a Bengalese finch female and a zebra finch female, both of whom had been reared by zebra finch parents. The test cage consisted of three compartments arranged in a line and separated by wire mesh. The choice females were housed in the lateral compartments $(30 \times 40 \times 40 \mathrm{~cm})$, the test male in the centre compartment $(60 \times 40 \times 40 \mathrm{~cm})$. The sides where the choice females were presented were changed each test to control for position preferences of the male birds. The males were exposed to a different female on the 1-h exposure and each of the three tests, respectively. The sexual preference of the males was scored according to the number of song phrases directed to one or other female, respectively. F rom the original values obtained during these tests, a preference score (PS) was calculated according to a formula also used by $K$ ruijt et al. (1983): $P S=Z f /(Z f+B f)$ where $Z f$ is the number of song phrases directed to the zebra finch female, and $B f$ is the number of song phrases directed to the Bengalese finch female. This score is zero if a bird sings exclusively to the Bengalese finch female, and is 1.00 when the bird sings exclusively to the zebra finch female. U ndirected singing did not occur in the test situation.

The 20 males of the first group reared by Bengalese finch foster parents were observed from day 20 (time of leaving the nest) to day 40 (time of isolation). Each group of siblings was monitored every second day from day 20 to day 40. The food trays were removed from the cages for $5 \mathrm{~h}$ preceding the observation period. Five minutes after replacing the trays, we started collecting data. Each sibling within a clutch was observed for 5 min by 'focal animal sampling' ( $M$ artin \& Bateson 1986). The sequence by which the birds were observed remained unchanged, but the observation was started with a different bird every day. A lthough other behaviour patterns were also recorded, begging and feeding events were given high priority, and here we report only the counts of 'feeding after begging' (Bischof \& Clayton 1991). 'F eeding' is used in this sense throughout the paper.

All males were also observed throughout the first and the second exposure to a female (a zebra finch female on day 100 and a Bengalese finch female on day 104, respectively). The frequencies of beak wiping (for a detailed description see M orris 1954) and pecking the perch, which can both be defined as displacement activities, were recorded as indicators of a bird's state of arousal (see Bindra 1959; M cF arland 1966; M aestripieri et al. 1992; and D iscussion).

In addition, we took blood samples from the males by puncturing the wing vein (vena subclavia) at $80,90,100$ and 104 days of age (see Fig. 1). On days 100 and 104, the blood samples were taken immediately after the 1-h exposure to a female. On days 80 and 90 the samples were taken after the males had spent $1 \mathrm{~h}$ under the same conditions (in the same observation room) as experienced on days 100 and 104, but without a female. To avoid effects of a possible diurnal rhythm in corticosterone secretion, all blood samples were taken at the same time in the morning. The blood plasma levels of corticosterone were determined by radioimmunoassay (for details see W ingfield \& F arner 1975; Pröve 1983). The mean recovery after extraction and chromatography was $55 \pm 0.09 \%$. The intra-assay variance was $19 \%$. R esults are shown for only 19 of 


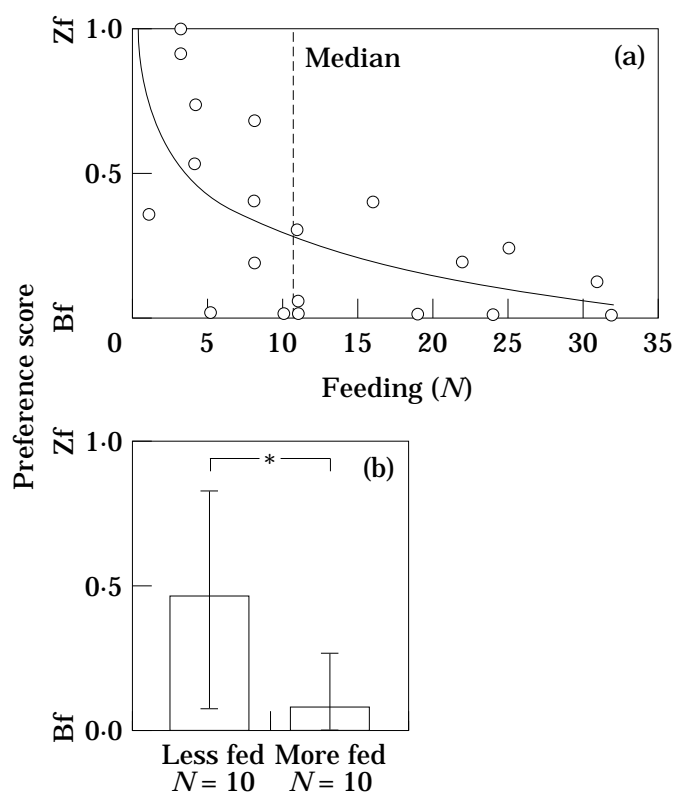

Figure 2. (a) R elation between the frequency of feeding by Bengalese finch foster parents and the preference scores across the three preference tests in experiment I $(\mathrm{N}=20)$. The median (broken line) was used to split the data into a group of 'less fed' and another group of 'more fed' birds (see text). (b) M edian, first and third quartile of the preference scores of males in the two groups 'less fed' and 'more fed'. $* P<0.05$ (see text).

the 20 males, because blood could not be sampled from one bird at one of the sampling points. Relative levels of hormone were used because the absolute levels of individual zebra finch males differed substantially, and we were interested in changes caused by the exposure to the female instead of absolute levels. For each bird, the mean of the absolute corticosterone levels on days 80 and 90 was defined as the base level. The corticosterone levels on days 100 and 104 were calculated as a percentage of this base level.

Differences between the two 'feeding' groups (see Fig. 2) were tested by a Mann-Whitney $\mathrm{U}$-test. $\mathrm{M}$ edians and first and third quartiles are used throughout the study to describe the results because the data were not normally distributed. Regression analyses and Spearman rank correlations were performed by the statistical package 'Wistat' on the A tari Mega ST 4.

\section{R esults}

F eeding

There was a clear negative relationship between the number of feeding bouts and the preference score indicating that the less the birds were fed by their Bengalese finch foster parents, the more likely they were to prefer zebra finches in later tests (Spearman's $r=-0.69, \mathrm{~N}=20, \mathrm{P}<0.01 ; \mathrm{Fig}$. 2a). A regression analysis showed that a nonlinear (logarithmic) regression had a higher coefficient and higher significance $(r=-0 \cdot 6, N=20$, $P=0.005)$ than a linear one $(r=-0.56, N=20$, $P=0.01)$. A lthough it is not appropriate to conclude that the relationship is really logarithmic, this shows that the decline of the regression is steeper in the first half of the graph. We therefore divided the sample into a group of the 10 less and the 10 more frequently fed birds, using the median as the separation line. The less frequently fed birds showed much more variation than the more fre quently fed ones, and the latter all expressed preference scores below 0.5 , thus tending towards a Bengalese finch preference ( $\mathrm{Fig}$. $2 \mathrm{~b}$ ). The medians in preference scores of both groups and the first and third quartiles were as follows: 'less fed' group: 0.46 (0.09, 0.82); 'more fed' group: 0.08 (0, $0 \cdot 27$ ). The medians differed significantly ( $M$ annWhitney $U$-test: $U=21 \cdot 5, P<0.05)$. We maintain this separation into two groups throughout the presentation of the remaining results.

\section{A rousal on day 100}

In the 'less fed' birds (Fig. 3) there was a positive relationship between the number of displacement activities at the time of exposure to the first (zebra finch) female (day 100) and the preference scores from the preference tests (Spearman's $r=0.63, \quad N=10, \quad P<0.05)$. The relative corticosterone levels of the 'less fed' birds were also positively related to their preference scores (Spearman's $r=0.62, N=10, P<0.05$ ).

Both findings can be interpreted as showing that the more a bird is aroused during its first exposure to a zebra finch female, the more likely it will prefer zebra finch females in the subsequent preference tests. This relationship cannot be seen in the 'more fed' birds ( $\mathrm{F}$ ig. $4 \mathrm{a}$, b). The differences in correlations between the 'less fed' and the 'more fed' groups are not due to differences in the median amounts of displacement activities or in 


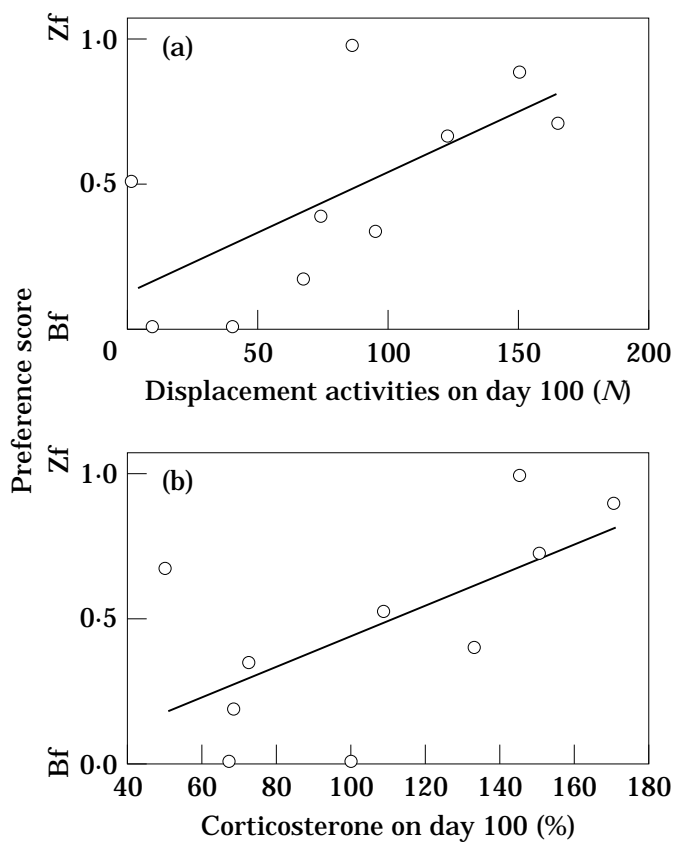

Figure 3. Relation between the preference scores of males $(\mathrm{N}=10)$ in the 'less fed' group and (a) displacement activities on day 100 and (b) relative corticosterone levels on day 100.

corticosterone levels. The medians in the relative levels of corticosterone of both groups and the first and third quartiles were as follows: 'less fed' group: 104 (68, 148); 'more fed' group: 102 (78, 141). The medians in displacement activities of both groups and the first and third quartiles are as follows: 'less fed' group: 80 (25, 136); 'more fed' group: $41(16,78)$. There was no significant difference between the measurements of the 'less fed' and the 'more fed' group in either parameter (Fig. 5).

\section{A rousal on day 104}

On day 104 there was no correlation between displacement activities and preference scores in either group; but, in contrast to the 100-day measurements, the correlation between relative corticosterone level on day 104 and preference score was significant in the 'more fed' birds (Spearman's $r=0.77, N=9, P<0.05 ; F i g .6 b$ ). In the 'less fed' birds, the correlation was not significant, but a trend in the same direction could be observed (Spearman's $r=0 \cdot 45, N=10$, Ns; Fig. 6a).

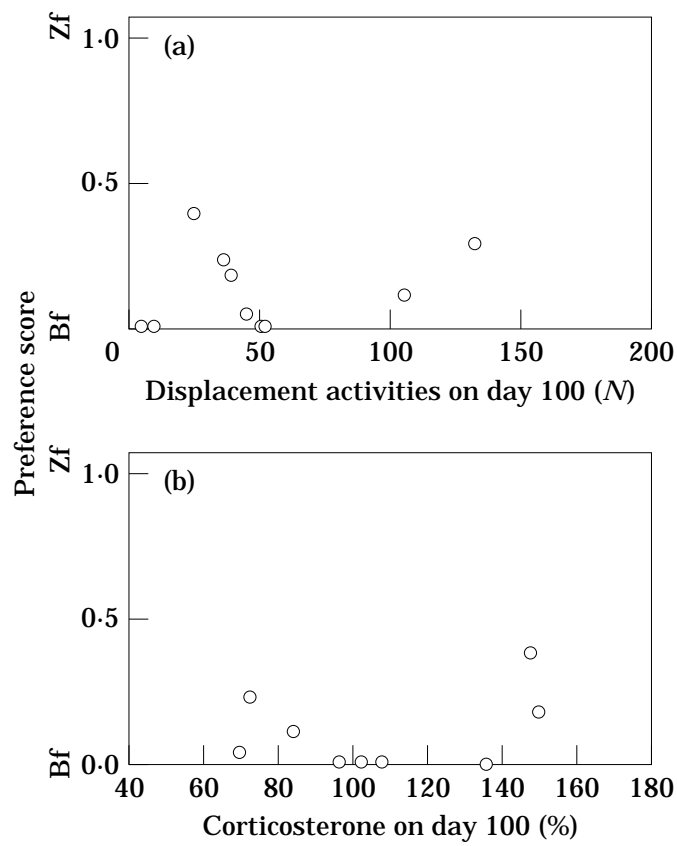

Figure 4. Relation between the preference scores of males in the 'more fed' group and (a) displacement activities on day $100(\mathrm{~N}=10)$ and (b) relative corticosterone levels on day $100(\mathrm{~N}=9)$.

U nexpectedly, the correlation was positive: the higher the corticosterone level, the more there was a trend towards a preference for zebra finch females, although the exposure was towards a Bengalese finch.

\section{D iscussion}

Our first experiment confirms the findings of previous studies (Bischof \& Clayton 1991; Immelmann et al. 1991; K ruijt \& M eeuwissen 1991) by demonstrating that under the given experimental conditions the preference of a male zebra finch can be altered even in adulthood. It also confirms and extends the results of Bischof \& Clayton (1991) concerning the factors involved in the development of the preferences. We found at least two factors that affect the preference score of a given male: first, the amount fed by the parents until 40 days of age, and second, the level of arousal induced by the 1-h exposure to the female on day 100.

Compared with previous studies which used similar designs (Bischof \& Clayton 1991; 

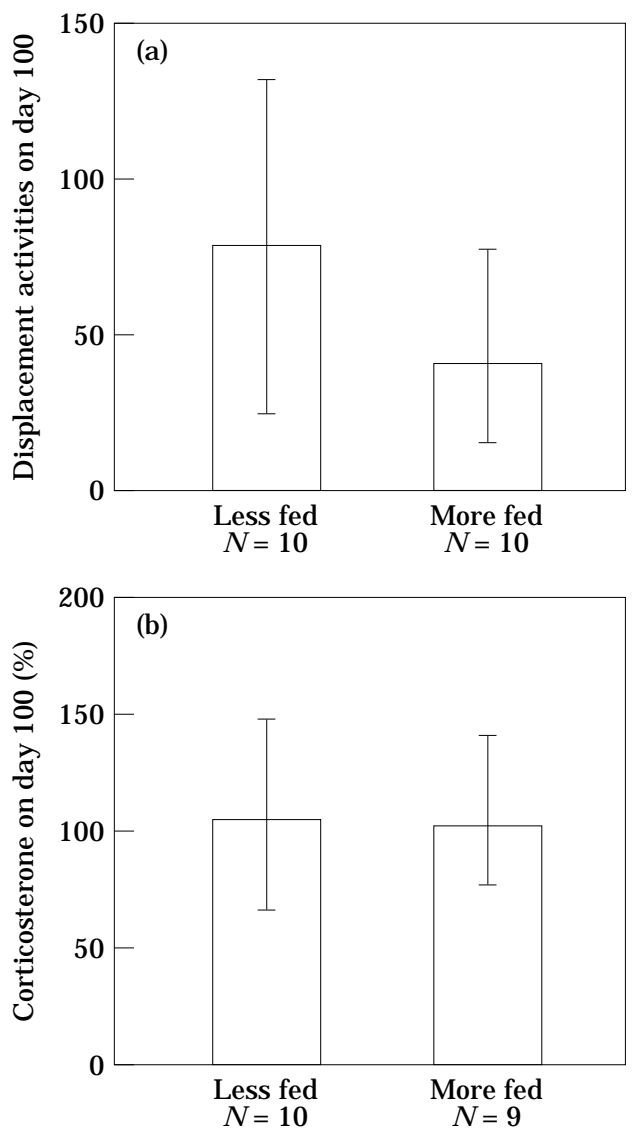

Figure 5. (a) F requency of displacement activities and (b) relative corticosterone levels of males of the 'less fed' and 'more fed' groups in experiment I on day 100.

Immelmann et al. 1991; K ruijt \& M eeuwissen 1991) our first experiment produced a large number of birds that retained their initial preference or became 'ditherers'. This may be due to the short time of exposure on day 100 (1 h) compared with the other studies (Bischof \& Clayton 1991: 1 week; K ruijt \& M eeuwissen 1991: 3 months; Immelmann et al. 1991: 7 months). 'D itherers' do not court exclusively to zebra finch or Bengalese finch females, respectively (K ruijt et al. 1983). Instead, they court to both species in varying percentages.

A ccording to ten $\mathrm{C}$ ate and co-workers (ten Cate 1986, 1987; Hollis et al. 1991) 'ditherers' develop a mixed or a double mental representation of objects which can be used for the selection of an appropriate sexual partner. When, as in our

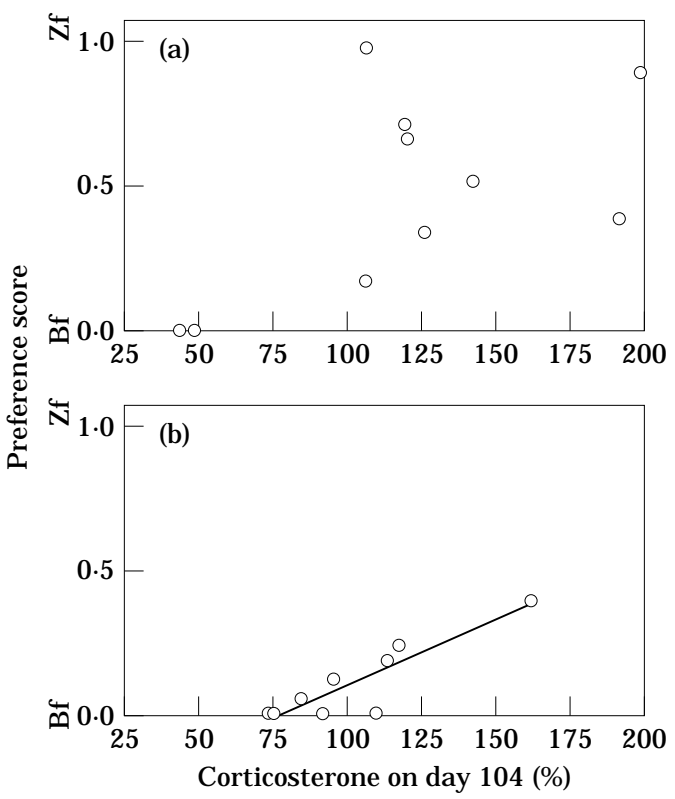

Figure 6. R elation between the relative corticosterone level on day 104 and the preference scores of males in (a) the 'less fed' group ( $N=10)$ and (b) the 'more fed' group $(\mathrm{N}=9)$ of experiment $\mathrm{I}$.

studies, the two exposures are separated by a substantial amount of time, it is more likely that a double instead of a mixed representation is developed (Chantrey 1974; H ollis et al. 1991). A ccording to the analysis-recognition-execution model (Bateson 1987, 1990, 1991) such representations are linked, in a second step, to an executive system which controls sexual behaviour. As Bateson claimed, access to this executive system is limited. Thus, if the first representation that is built captures a large number of the accessible links, there is not much left for a second representation. When no more links are open, the imprinting process is finished and the preference of the bird is no longer alterable. The validity of such a model has been shown for example by Bolhuis \& Bateson (1990) for filial imprinting.

The results of our first experiment may be explained by the same model. The birds of the 'more fed' group showed either an absolute or a relative preference for the Bengalese finch, the preference scores ranging between 0 and $0 \cdot 4$. Even a high arousal level at the time of the exposure to the zebra finch female, which led to more zebra finch directed preference scores in the 'less fed' group, did not have any effect in the 'more fed' 
group. In terms of the above-mentioned model, this means that most of the accessible links to the executive system were al ready captured by the first (Bengalese finch) representation. Thus the zebra finch representation, which was built during the second exposure, could not get sufficient influence on the executive system for sexual behaviour.

In the birds of the 'less fed' group, the Bengalese finch representation was obviously less effective in occupying the links to the executive system, allowing the second (zebra finch) representation to capture substantial numbers of the accessible links. Consequently, these birds could develop a stronger preference for the zebra finch female. However, if the preference score in some way reflects the balance of the access of the two representations to the executive system, the development of the representations was very different for each bird of the 'less fed' group.

What are the factors that affect the establishment of a preference? $M$ any previous studies have shown that time, sequence and duration of exposure are important parameters (Bateson 1981; Immelmann \& Suomi 1981; Bolhuis \& Bateson 1990). A comparison of our results with the above-mentioned studies (Bischof $\&$ Clayton 1991; Immelmann et al. 1991; K ruijt \& $M$ eeuwissen 1991) also indicates that the longer the exposure to a zebra finch female after 100 days, the more the zebra finch female preference is developed. Because we held exposure times constant, they could not cause the observed differences. Instead, our results indicate that the 'quality' and the context of presentation of the stimulus are also of major importance. The learning about the Bengalese finch parents correlates positively with feeding during development, and learning about the zebra finch female correlates with the arousal level induced by the first exposure. That the latter effect could be seen only in the 'less fed' group may arise from a ceiling effect. Probably, the information from the two sources of early and adult experience must be balanced to a certain degree. If one of the sources is dominating, as may be the case in the 'more fed' group, the effects of the other may be obscured.

It may be argued that the feeding we observed between days 20 and 40 might be not the cause of the preference, but an indicator of a previously developed preference. We cannot exclude this but ten Cate (1984) has shown that interaction between parents and offspring is important for the development of a preference. Because feeding is the main interaction before the young zebra finches leave their nest, it is plausible that this behaviour is indeed the main factor that causes the preference. Moreover, it is also plausible to presume that, if there is indeed a previously acquired preference, this is further enhanced by feeding after fledging.

At present, we can only speculate which mechanisms underlie these enhancements of learning. L earning while being fed may represent some sort of conditioning, as has been suggested for filial and sexual imprinting (reviewed by Bolhuis 1991), or has been shown to be important for song learning (A dret 1993). Being fed may also simply enhance the level of arousal, similar to the situation where the young male is exposed to a female for the first time after isolation. Thus, the simplest explanation which probably applies for both, the exposure during feeding and to the female at 100 days, is that the more frequently or strongly the bird is aroused, the more information is acquired. It is important, however, to state here that the two explanations are not mutually exclusive.

One could also argue that our measures of 'arousal' are actually measures of conflict. Thus, for the birds that showed high measures of corticosterone or displacement activities, the exposure to the novel stimulus may be more conflicting than for those with low measures. Conflicting situations, however, also result in high arousal (e.g. Bindra 1959; M cF arland 1966; M aestripieri et al. 1992). M ale zebra finches, in exactly the same experimental design as used in our study, show high activities in certain brain areas, when exposed to the female after the isolation period (Bischof \& Herrmann 1986, 1988); this activity represents physiological arousal. Other studies indicate that the activated brain areas are involved in imprinting (Wallhäusser \& Scheich 1987; Rollenhagen \& Bischof 1991, 1994). We are thus quite confident that arousal is the decisive factor for the determination of the amount of learning. However, the possibility of a contribution of the factor 'conflict' cannot be fully excluded.

The second exposure to a Bengalese finch female at 104 days was mainly included to make our results comparable to the above-mentioned other studies of similar design. The previous studies, however, have shown that at this time the birds are already stably imprinted. No new change of preference can be induced by this second 
exposure. Thus, a correlation between preference scores and displacement activities was not to be expected in this situation. A fter the exposure to the female, however, there was a strong, positive correlation between corticosterone level and preference scores for the 'more fed' group and the same tendency for the 'less fed' group. This means, the more the preference of a given bird was zebra finch directed, the higher was its corticosterone level. This result is difficult to explain. Probably, an extreme preference score, which has been consolidated in the course of the first exposure, also means a strong narrowing of preferences and thus strong avoidance of non-familiar objects. The corticosterone level in this situation would then probably be indicative of the fear induced in the birds by the exposure to the non-familiar object (e.g. M artin \& Schutz 1975).

\section{EXPERIMENT II}

Our results so far can be discussed in terms of Bateson's (1987, 1990, 1991) model which claims that the representations that are built are linked to an executive system, and no new representations can be connected to an executive system if the free links are exhausted. One argument, however, suggested that the story may not be so simple. As mentioned earlier, thinking about sexual imprinting as a two-stage process avoids the question of how the immature bird 'knows' what the appropriate features of a prospective sexual partner may be (Bischof 1994). Thus, the internal representation of the parent(s), which obviously has a strong impact on the sexual preference expressed in adulthood, is probably not linked to the executive system at the time of its generation. This is done, according to the 'two-stage' idea, by the 'consolidation' process which occurs when the young bird comes into a strongly arousing situation for the first time in its life. We have learnt from our first experiment, however, that during the first sexual encounter of the male a new representation (that of a zebra finch female) can be established, if the bird had less contact (feeding) with its foster parents. It could therefore be argued that, according to Bateson's model, the consolidation of the preferences occurs simply because the new representation captures the still accessible links. The 'two-stage' idea, in addition, would claim that at this time two processes may occur simultaneously: first, the installation of a new representation, and second, the consolidation process linking the two representations to the executive system. To support this idea of two independent processes, one has to find a situation by which only one of the two processes takes place. Our second experiment shows how the proposed system reacts in such a situation.

\section{M ethods}

R earing and testing conditions in experiment II were identical to those of experiment I. The only difference was that a nestbox $(14 \times 14 \times 17 \mathrm{~cm})$ was attached to the home cage on the last day of the isolation period, day 99. The experimental group could gain access to the interior of the nestbox, where a nest was built from coir by the experimenter. After $24 \mathrm{~h}$, on day 100 , a zebra finch female was put into the cage for $1 \mathrm{~h}$. Thereafter the female and the nestbox were removed. On day 104, the second exposure to a Bengalese finch female was performed without a nestbox.

The birds of a control group were given a nestbox on the same day. This nest, however, was attached to the cage by the rear side, so that the animals had no access to the interior and probably could not identify the box as a place for breeding. The males were also exposed to a zebra finch female for $1 \mathrm{~h}, 24 \mathrm{~h}$ later. For the exposure the nestbox was turned round, the entrance exposed. Following the exposure, the nestbox was removed together with the zebra finch female. On day 104, these birds were also exposed to a Bengalese finch female without a nestbox for $1 \mathrm{~h}$. Preference scores were determined as described for experiment $\mathrm{I}$.

\section{R esults}

A fter being exposed to the nestbox, the males did not acquire any new information about the zebra finch female which they saw the next day. In the preference tests, all males of this group sang exclusively to the Bengalese finch female, all individual scores being zero ( $\mathrm{F}$ ig. 7a). In contrast, the males of the control group, which could see only the rear of the nestbox, varied in preference scores comparable to the birds in experiment I (Fig. 7b). The two groups differed significantly ( $M$ ann-W hitney $U$-test: $U=15, P<0.01$ ). 


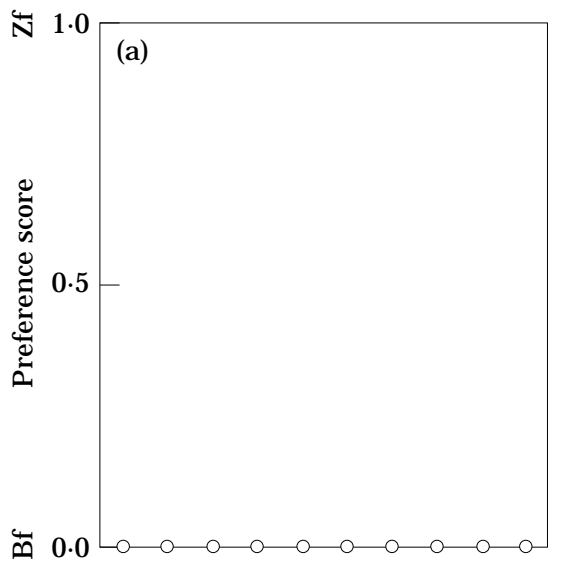

Nestbox

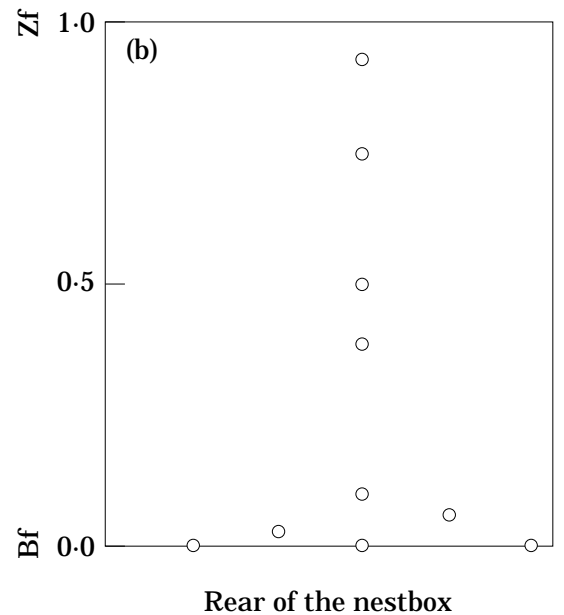

Figure 7. Individual preference scores of males in (a) the 'nestbox' group and (b) the control/'rear of the nestbox' group of experiement II. X-scales are arbitrary.

\section{D iscussion}

This result clearly indicates that seeing a nestbox after some time of isolation consolidates the sexual preference of a male zebra finch. Earlier studies (Bischof \& Clayton 1991; Immelmann et al. 1991; K ruijt \& M eeuwissen 1991) have shown that direct interaction with a female is not necessary for consolidation; exposure to a female separated by wire has the same effect as exposure within the same cage ( $\mathrm{K}$ ruijt \& M eeuwissen 1991). Our experiment shows that even the appearance of a female is not necessary for consolidation to occur. Thus, it demonstrates that consolidation is really independent of the construction of a representation.

At present, we can only speculate about the factor inducing the consolidation process that led to an exclusive preference for the Bengalese finch female in the 'nestbox' group. B ecause the control group received a box of the same size, but without access to the nest, we are confident that the effect was due to the birds' recognition of the open box as a nesting place and not to some unspecific stimulation, induced for example by the installation of the box, or by the shadow it threw into the cage. Probably, the view of the nestbox brought the young male into a condition of sexual arousal, as the young male was aroused by the zebra finch female in experiment I. Behavioural observations and physiological measurements have to be performed to support this idea. It seems plausible, however, that owing to the presentation of the nest the machinery for the execution of sexual behaviour was started. Because up to this time no representation of an appropriate object for courtship was coupled to the executive system, memory was searched for such an object, and the representation of the parents' species (or even the mother) was coupled to the executive system.

\section{GENERAL DISCUSSION}

Taken together, our experiments show that during exposure to a zebra finch female at 100 days of age (experiment I) two processes occur simultaneously: the acquisition of a new representation and the consolidation of the sexual preference. These two processes are, principally, independent of each other, as experiment II demonstrates. The results therefore support the idea of imprinting as a two-stage process, although, under certain circumstances, the two stages can overlap in time. This suggests that neither is a 'single shot' event, and that consolidation is probably slower than acquisition, because otherwise our birds in experiment I would not have developed any substantial preferences for the zebra finch female. It is not possible at present to speculate about the underlying physiological machinery. A rousal mechanisms are probably involved in both acquisition and consolidation. At present it is not possible to 
distinguish between general arousal and arousal linked to some motivational system such as courtship or breeding and of different quality in both stages. Further behavioural and physiological studies may throw light on this problem.

It is also unclear whether seeing a nest is a consolidating event under natural conditions. To answer this question detailed knowledge about the development of the zebra finch under natural conditions would be necessary. However, if we take into account that seeing a female also consolidates the preference then it seems more likely that there may be a variety of stimuli that arouse the bird and consolidate previously acquired preferences. One has also to take into account that under normal conditions the first sexual encounter, or the first situation with sexual arousal, occurs much earlier, and that the arousal level in the wild may not be as high as in our experiments, where the birds were isolated for a long time before they were exposed for the first time to the female. This may mean that linking representations to the executive system of sexual behaviour starts much earlier, possibly at about 30-35 days, when the first courtship events are observed (K alberlah 1980), and consolidation may, under natural conditions, take place in small portions over a long time. If the experience with the parents (or the mother) is not sufficient for building an adequate representation, information from other sources such as siblings or unfamiliar birds may also be used. A first indication that this view may be correct comes from a study by ten Cate et al. (1993), which indicates that a young zebra finch learns about its prospective sexual partner between days 30 and 60 , in contrast to many studies performed earlier. If this finding turns out to be robust, it supports the idea that in the 'classical' sensitive phase in early development a social preference is built, which guides the construction of the later developing sexual preference; in other words, it supports the idea of sexual imprinting as a two-stage process.

Our results shed some light on the processes involved in the construction of the preference. Interaction with the parents, as indicated by our 'feeding' results, are important for building the social preference. The sexual preference, in contrast, is not dependent on interactions, but is established by enhanced arousal. Whether this is arousal in a sexual context or, as some of our results suggest, may be more general, has still to be examined. L ast but not least, our experiments provide evidence that the terms 'modification' and 'consolidation', suggested by Kruijt \& M eeuwissen (1991) to indicate whether the pre viously acquired preference is modified or consolidated during the first exposure to a female, do not represent opposing processes. The first process, the acquisition of new features to establish a representation, and the second process of linking a representation to sexual behaviour, may overlap in time, but can also, at least in an appropriately designed experiment, be separated from each other.

\section{ACKNOWLEDGMENTS}

We gratefully acknowledge the comments of A. Turner, G. de Vos and J. J. Bolhuis, which substantially improved the paper. Our thanks are also due to $\mathrm{N}$. Clayton for correcting the English text. The study was supported by the Deutsche F orschungsgemeinschaft (Bi 245/9-1).

\section{REFERE NCES}

A dret, P. 1993. O perant conditioning, song learning and imprinting to taped song in the zebra finch. A nim. B ehav., 46, 149-159.

Bateson, P. P. G. 1966. The characteristics and context of imprinting. Biol. Rev., 41, 177-220.

Bateson, P. P. G. 1981. Control of sensitivity to the environment during development. In: Behavioral Development. The Bielefeld Interdisciplinary Project (Ed. by K . Immelmann, G. W. Barlow, L. Petrinovich \& M. M ain), pp. 432-453. Cambridge: Cambridge U niversity Press.

Bateson, P. P. G. 1987. Imprinting as a process of competitive exclusion. In: Imprinting and Cortical Plasticity (Ed. by J. P. Rauschecker \& P. M arler), pp. 151-168. N ew Y ork: J ohn Wiley.

Bateson, P. P. G. 1990. Is imprinting such a special case? P hil. Trans. R. Soc. Ser. B, 329, 125-131.

Bateson, P. P. G. 1991. A re there principles of behavioural development? In: The Development and Integration of Behaviour (Ed. by P. Bateson), pp. 19-39. Cambridge: Cambridge U niversity Press.

Bindra, D. 1959. M otivation: A Systematic Reinterpretation. $\mathrm{N}$ ew $\mathrm{Y}$ ork: R onald Press.

Bischof, H.-J. 1994. Sexual imprinting as a two-stage process. In: Causal Mechanisms of Behavioural Development ( $\mathrm{E}$ d. by J. A. Hogan \& J. J. Bolhuis), pp. 82-97. Cambridge: Cambridge U niversity Press.

Bischof, H.-J.\& Clayton, N. 1991. Stabilization of sexual preferences by sexual experience in male zebra finches (Taeniopygia guttata castanotis). Behaviour, 118, 144-155. 
Bischof, H.-J . \& H errmann, K . 1986. A rousal enhances 14-C-2 deoxyglucose uptake in four forebrain areas of the zebra finch. Behav. Brain Res., 21, 215-221.

Bischof, H.-. \& \& Herrmann, K. 1988. Isolationdependent enhancement of 2-(14C)-deoxyglucose uptake in the forebrain of zebra finch males. Behavl neural Biol., 49, 386-397.

Bolhuis, J. J. 1991. M echanisms of avian imprinting: a review. Biol. Rev., 66, 303-345.

Bolhuis, J. J. \& Bateson, P. 1990. The importance of being first: a primacy effect in filial imprinting. A nim. Behav., 40, 472-483.

Chantrey, D. F. 1974. Stimulus preexposure and discrimination learning by domestic chicks: effect of varying interstimulus time. J . comp. physiol. P sychol. 87, 517-525.

Hess, E. H. 1973. Imprinting: Early Experience and the Developmental Psychobiology of Attachment. N ew Y ork: Van N ostrand R einhold.

H ollis, K . L., ten Cate, C.\& Bateson, P. 1991. Stimulus representation: a subprocess of imprinting and conditioning. J . comp. P sychol., 105, 307-317.

Immelmann, K. 1972a. Sexual and other long-term aspects of imprinting in birds and other species. In: Advances in the Study of Behaviour, Vol. 4 (Ed. by D. S. Lehrmann, R. A. Hinde \& E. Shaw), pp. 147-174. N ew Y ork: A cademic Press.

I mmelmann, K . 1972b. The influence of early experience upon the development of social behaviour in estrildine finches. In: Proceedings of the XV International Ornithological Congress D en H aag 1970 (Ed. by K . K Voous), pp. 316-338. Leiden: E. J. Brill.

Immelmann, K. 1985. Sexual imprinting in zebra finches: mechanisms and biological significance. Proc. int. ornithol. Congr., X VIII, 156-172.

Immelmann, K ., Pröve, R ., Lassek, R . \& Bischof, H.-J. 1991. Influence of adult courtship experience on the development of sexual preferences in zebra finch males. A nim. Behav., 42, 83-90.

Immelmann, K . \& Suomi, S. J. 1981. Sensitive phases in development. In: Behavioral Development. The Bielefeld Interdisciplinary Project (Ed. by $\mathrm{K}$. Immelmann, G. W. Barlow, L. Petrinovich \& M. M ain), pp. 395-431. Cambridge: Cambridge U niversity Press.

K alberlah, H. H. 1980. Quantitative U ntersuchungen zur Ontogenese des Sexualverhaltens beim Zebrafinken (Taeniopygia guttata castanotis). Ph.D. thesis, U niversity of Bielefeld.

K ruijt, J. P. \& M eeuwissen, G. B. 1991. Sexual preferences of male zebra finches: effects of early and adult experience. A nim. B ehav., 42, 91-102.

K ruijt, J. P. \& M eeuwissen, G. B. 1993. Consolidation and modification of sexual preferences in adult male zebra finches. N eth. J. Z ool., 43, 68-79.
K ruijt, J. P., ten Cate, C. J . \& M eeuwissen, G. B. 1983. The influences of siblings on the development of sexual preferences of male zebra finches. Devl P sychobiol., 16, 233-239.

M CF arland, D. 1966. On the causal and functional significance of displacement activities. Z. T ierpsychol., 23, 217-235

M aestripieri, D., Schino, G., A ureli, F. \& Troisi, A . 1992. A modest proposal: displacement activities as an indicator of emotions in primates. A nim. Behav., 44, 967-979.

M artin, J. T. \& Schutz, F. 1975. A rousal and temporal factors in imprinting in mallards. D evl Psychobiol., 7, 69-78.

M artin, P. \& Bateson, P. 1986. M easuring B ehaviour: an Introductory Guide. Cambridge: Cambridge U niversity Press.

M orris, D. 1954. The reproductive behaviour of the zebra finch (Poephila guttata), with special reference to pseudofemale behaviour and displacement activities. B ehaviour, 6, 271-321.

Pröve, E. 1983. Hormonal correlates of behavioural development in male zebra finches. In: H ormones and B ehaviour in $\mathrm{H}$ igher $\mathrm{V}$ ertebrates ( $\mathrm{Ed}$. by J. Balthazart, E. Pröve \& R. Gilles), pp. 368-374. Berlin: SpringerVerlag.

Rollenhagen, A.\& Bischof, H. J. 1991. Rearing conditions affect neuron morphology in a telencephalic area of the zebra finch. N euroR eport, 2, 711-714.

Rollenhagen, A. \& Bischof, H. J. 1994. Phase specific morphological changes indicated by social experience in two forebrain areas of the zebra finch. B ehav. Brain Res., 65, 83-88.

ten Cate, C. 1984. The influence of social relations on the development of species recognition in zebra finch males. B ehaviour, 91, 263-285.

ten Cate, C. 1986. Sexual preferences in zebra finch males raised by two species: I. A case of double imprinting. J. comp. P sychol., 100, 248-252.

ten Cate, C. 1987. Sexual preferences in zebra finch males raised by two species: II. The internal representation resulting from double imprinting. Anim. Behav., 35, 321-330.

ten Cate, C., Vos, D. R. \& M ann, N. 1993. Sexual imprinting and song learning: two of one kind? $N$ eth. J. Z ool., 43, 34-45.

Wallhäusser, E. \& Scheich, H. 1987. A uditory imprinting leads to differential 2-deoxyglucose uptake and dendritic spine loss in the chick rostral forebrain. Devel. Brain Res., 31, 29-44.

W ingfield, J . C . \& F arner, D. S. 1975. The determination of five steroids in avian plasma by radioimmunoassay and competitive protein-binding. Steroids, 26, 311-327. 
